\title{
TDP 1 inhibitors as potential antitumor drugs
}

\author{
A. Zakharenko ${ }^{1 *}$, O. Luzina ${ }^{2}$, O. Salomatina ${ }^{2}$, O. Zakharova ${ }^{1}$, K. Volcho ${ }^{2}$, \\ V. Kaledin ${ }^{3}$, V. Nikolin ${ }^{3}$, N. Popova ${ }^{3}$, N. Salakhutdinov ${ }^{2}$, O. Lavrik ${ }^{1}$ \\ ${ }^{1}$ Institute of Chemical Biology and Fundamental Medicine SB RAS, Novosibirsk, Russia \\ ${ }^{2}$ Institute of Organic Chemistry SB RAS, Novosibirsk, Russia \\ ${ }^{3}$ Institute of Cytology and Genetics SB RAS, Novosibirsk, Russia \\ *e-mail:sashaz@niboch.nsc.ru
}

Key words: tyrosyl-DNA polymerase 1 inhibitors, usnic acid, bile acids

Motivation and Aim: Tyrosyl-DNA-phosphodiesterase 1 (Tdp1) is promising target for antitumor therapy based on damage of tumor DNA, induced by topoisomerase 1 (Top1) inhibitors such as camptothecin and its clinical derivatives [1]. Thus, inhibition of Tdp1 may enhance therapeutic effect of Top1 inhibitors, sensitizing tumor cells to their action [2]. The objectives of the study were: search of effective Tdp1 inhibitors among derivatives of the natural biologically active compounds usnic and bile acids; definition for the most effective inhibitors their own cytotoxicity against tumor cell lines and their influence on the cytotoxic effect of camptothecins; study of the influence of the most promising compounds on the tumor growth and metastasis of Lewis carcinoma in vivo. Methods and Algorithms: The inhibitory properties of compounds were determined using real-time detection of Tdp1 activity [3]. Cytotoxicity of compounds along and in combination with camptothecin derivative topotecan was determined using a standard MTT test for tumor cell lines. For in vivo experiments, mice C57BL/6 were used with Lewis carcinoma metastasizing into the lungs.

Results: In vitro studies have established high inhibitory activity of usnic acid derivatives against $\mathrm{Tdp} 1$ (half inhibitory concentrations $\mathrm{IC}_{50}$ were in the range of 20 to $150 \mathrm{nM}$ ). Also we have found a new class of Tdp1 inhibitors based on the bile acid scaffold with $\mathrm{IC}_{50}$ up to $0.29 \mu \mathrm{M}$. In combination with topotecan, some of usnic acid derivatives increase the cytotoxic effect of the former from 2 to 12 times, being used in the non-toxic concentrations. In vivo studies have shown that under the influence of the usnic acid derivatives, a $30 \%$ decrease in the volume of the primary tumor and at least two-fold enhancement of the antimetastatic effect of topotecan on Lewis carcinoma take place.

Conclusion: The use of the Tdp 1 inhibitors made it possible to unequivocally demonstrate increasing influence on the antitumor effect of topotecan in relation to the transplanted tumor of mice, both on its primary grafts and on distant metastases.

Acknowledgements: Supported by Russian Science Foundation (grant No. 16-13-10074).

\section{References}

1. Comeaux E.Q., van Waardenburg R.C. (2014) Tyrosyl-DNA phosphodiesterase I resolves both naturally and chemically induced DNA adducts and its potential as a therapeutic target. Drug Metab. Rev. 46(4): 494-507.

2. Kawale A.S., Povirk L.F. (2018) Tyrosyl-DNA phosphodiesterases: rescuing the genome from the risks of relaxation. Nucleic Acids Res. 46(2):520-537.

3. Zakharenko A. et al. (2015) Synthesis and biological evaluation of novel tyrosyl-DNA phosphodiesterase 1 inhibitors with a benzopentathiepine moiety. Bioorg. Med. Chem. 23(9):2044-2052. 